\title{
Periodic orbits in tall laterally heated rectangular cavities
}

\author{
Marta Net* and Juan Sánchez Umbría ${ }^{\dagger}$ \\ Departament de Física, Universitat Politècnica de Catalunya. \\ Jordi Girona Salgado 1-3. Campus Nord. Mòdul B4. 08034 Barcelona. Spain
}

(Dated: January 12, 2017)

\begin{abstract}
This study elucidates the origin of the multiplicity of stable oscillatory flows detected by time integration in tall rectangular cavities heated from the side. By using continuation techniques for periodic orbits, it is shown that initially unstable branches, arising at Hopf bifurcations of the basic steady flow, become stable after crossing Neimark-Sacker points. There are no saddlenode or pitchfork bifurcations of periodic orbits, which could have been alternative mechanisms of stabilization. According to the symmetries of the system, the orbits are either fixed cycles, which retain at any time the center-symmetry of the steady flow, or symmetric cycles involving a time shift in the global invariance of the orbit. The bifurcation points along the branches of periodic flows are determined. By using time integrations, with unstable periodic solutions as initial conditions, it is found out which of the bifurcations at the limits of the intervals of stable periodic orbits are sub or supercritical.
\end{abstract}

PACS numbers: 47.15.-x, 47.20.-k

Keywords: Thermal convection, Periodic flows, Stability

\section{INTRODUCTION}

Thermal convection in enclosed cavities with either vertical or horizontal heating has long been studied because of its relevance in industrial applications, for instance, in the successful liquid encapsulated growth of single crystals [1]. Convective motions must be damped in order to maintain the melt as steady as possible and avoid the growth of perturbations leading to transitions to time dependent flows [2]. Other examples of the interest of this problem are the design of large-scale laser systems in order to minimize the optical distortion due to the buoyancy-driven flow [3], or the optimal heating/cooling and isolation of buildings.

The initial convective flow, in rectangular laterally heated cavities, consists in a single steady vortex, which fills the domain. The first instability of this flow breaks its center-symmetry for any aspect ratio, $\Gamma$ (defined in this work as the ratio of the height of the box, $h$, to the width, $d$ ). However the source of instability leading to time periodic dynamics is different depending on this parameter, so the frequencies of the bifurcated solutions are also very different. The first oscillatory motions, in the case of air (Prandtl number $\operatorname{Pr}=0.71$ ) and $1 \leqslant \Gamma \leqslant 3$, are due to the instability of the detached flow from the boundary layers near the corners of the box. It gives rise to internal waves. In contrast, for $4 \leqslant \Gamma \leqslant 8$ the first bifurcation of the steady state leads to the appearance of traveling waves in the boundary layers with frequencies ten times higher than those of the internal waves. In the range $2.8 \lesssim \Gamma \lesssim 4$ the first branch of steady solutions is double-folded and both types of time-dependent dynamics can coexist. For more details see [4] and references

\footnotetext{
*marta.net@upc.edu

$\dagger$ juan.j.sanchez@upc.edu
}

therein.

The transition from the steady flow to periodic solutions has been studied by several authors, mainly for $\Gamma<8$, either by using time integration, as for instance in Ref. [5], or by computing the stability of the fixed points obtained by continuation methods (see [4, 6-9], among others). In Ref. [6] an enclosure of $\Gamma=1 / 4$ filled with a liquid metal was considered, and the influence of different boundary conditions and $P r$ on the onset of the oscillations was analyzed. A thorough description of the steady states of the convective flows of low $\mathrm{Pr}$, for $1 / 11<\Gamma<1$, including the stability regions, was given in Ref. [7]. On the other hand, three-dimensional computations of the first instability were presented in Ref. [9]. Several boxes of $1 / 5<\Gamma<1 / 2$ and $1 / 6<\Gamma_{y}<1$, and Prandtl numbers in the range $10^{-7}<\operatorname{Pr}<0.03$ were considered. The aspect ratios were defined as $\Gamma=h / d$ and $\Gamma_{y}=h / L_{y}$, $L_{y}$ being the depth in the transverse direction. It was shown that, at low $\mathrm{Pr}$, the type of transition (steady or oscillatory) depends on the size of the box, and on the symmetries broken at the bifurcation.

Some direct numerical simulations analyzing the routes from periodic flows to chaos have been published recently. A complete description of the transition to turbulence is given in Ref. [10] for air-filled $2 \mathrm{D}$ containers of $\Gamma=2$, under a dynamical systems point of view. Two main routes that gradually break the symmetries of the solutions as sets were found. Finally, for Rayleigh numbers higher than $10^{8}$ the two paths collide, and a single hyperchaotic attractor with restored global symmetry is attained. The three-dimensional dynamics of air between two vertical parallel planes at distance $d$, with spatial periods $h$ and $L_{y}$ in height and depth, respectively, was studied in Ref. [11] for $\Gamma_{y}=L_{y} / d=1$ and $\Gamma=h / d=2.5$. It was found that, as the Rayleigh number, $R a$, was increased, a sequence of period-doubling bifurcations led to chaos. An intermittent orbit visiting two unstable sym- 
metric solutions was detected for higher $R a$.

Numerical simulations in tall laterally heated rectangular domains showed the coexistence of various branches of periodic orbits $(\mathrm{PO})$, which origin was guessed in Ref. [4] from the comparison of the critical eigenfunction of the steady solutions at the bifurcating points with the spatial and temporal structure of the periodic orbits. The present paper focuses on the computation of the branches of periodic flows by continuation methods, and the location of the limits of their regions of stability by computing the leading Floquet multipliers. For this purpose a cavity of $\Gamma=8$ filled with air is considered. This problem was proposed as a benchmark to test different time integration codes in the First MIT Conference on Computational Fluid and Solid Dynamics in June 2001. The contributions by several authors were published in a special issue of Int. J. Numer. Meth. Fluids (see [3, 8, 12-15], among others). To compare the results several point data, temporal and spatial time averages, and fluctuations with respect to the mean values, of the velocity, vorticity, pressure differences and temperature were supplied by the contributors, in addition to the period, mean heat flux transport on the lateral boundaries, or a metric to measure the loss of the center-symmetry of the temperature field, although the full description of the solutions of this problem remained open. For example, it was not clear if the multiple stable PO appear after the stabilization of unstable ones via saddle-node or symmetry-breaking bifurcations.

The physical interest of the 2D approximation is not worthless. As shown in Ref. [16] the internal core of the fluid remains, in general, almost stably stratified. The onset of time-dependent flows is bidimensional for boxes of large aspect ratios if there is enough space in the periodic transverse direction, and nearly $2 \mathrm{D}$ when $\Gamma<7$. For instance, in the latter case, and enforcing transverse periodicity, only very low amplitude and frequency structures travel in this direction before the boundary layer instability arises.

Continuation methods have several main advantages versus more usual time-dependent simulations. The critical parameters corresponding to the threshold are only affected by the truncation error. They are obtained faster than if they are inferred from the transients of the time integrations, specially when oscillatory flows are involved. Another advantage is that the computation of unstable branches of orbits allows to understand more easily the transients among branches and the origin of some stable flows found by time evolution.

The remaining of the paper is organized as follows. The equations, their numerical treatment, and the testing of the codes are discussed in Sec. II. Sec. III contains a brief revision of the stability of the basic steady solution and of the symmetries of the eigenfunctions, extending previous results. The periodic flows bifurcated from the branch of steady solutions, and computed by means of continuation methods, are described in Sec. IV. Their stability is analyzed in the same section determining the bifurcation points where quasiperiodic flows arise. Some characteristics of the two and three frequency solutions are also studied. The paper concludes in Sec.V with a summary of the results obtained.

\section{MATHEMATICAL FORMULATION}

The thermal convection of a fluid of Prandtl number $\operatorname{Pr}=0.71$, filling a rectangular cavity, $\Omega$, of aspect ratio $\Gamma=8$, with a non-slip boundary is studied. The side walls are maintained at uniform temperatures, the left higher than the right. The top and bottom walls are insulating, and the fluid is subject to a vertical gravity $\mathbf{g}=-g \mathbf{j}, \mathbf{j}$ being the unit vector pointing upwards.

The Boussinesq approximation of the mass, momentum and energy equations is used. They are nondimensionalized by taking the width of the box, $d$, the difference of temperature between the left and right sides, $\Delta \mathcal{T}>0$, and $d^{2} / \kappa, \kappa$ being the thermal diffusivity, as distance, temperature and thermal time scales, respectively. In non-dimensional units $\Omega=[0,1] \times[0, \Gamma]$.

Let $x$ and $y$ be the horizontal and vertical coordinates, respectively. The system is written by splitting the temperature as $\mathcal{T}(x, y, t)=(1-x)+\Theta(x, y, t)$, in order of having homogeneous boundary conditions on the vertical walls. It follows that

$$
\begin{aligned}
& \nabla \cdot \mathbf{v}=0 \\
& \left(\partial_{t}+\mathbf{v} \cdot \boldsymbol{\nabla}\right) \mathbf{v}=-\nabla \pi+\operatorname{Pr} \Delta \mathbf{v}+\operatorname{Ra} \operatorname{Pr}(-x+\Theta) \hat{y}, \\
& \left(\partial_{t}+\mathbf{v} \cdot \boldsymbol{\nabla}\right) \Theta=\Delta \Theta+u
\end{aligned}
$$

where $\mathbf{v}=(u, v)$ is the velocity field, and the gradient of $\pi=\left(p / \rho_{0}\right)+\left(1+\alpha \mathcal{T}_{0}-\alpha \Delta \mathcal{T} d^{3} / \kappa^{2}\right) g y$ gives the modified pressure term, which includes two terms coming from the Boussinesq approximation, and the last from the splitting of the temperature. The constant $\alpha$ is the thermal expansion coefficient, and $\rho_{0}$ is the constant density at the reference temperature $\mathcal{T}_{0}$.

The problem depends on the Rayleigh and Prandtl numbers defined as

$$
R a=\frac{\alpha g \Delta \mathcal{T} d^{3}}{\kappa \nu} \quad \text { and } \quad \operatorname{Pr}=\frac{\nu}{\kappa},
$$

where $\nu$ is the kinematic viscosity. The former, which is proportional to the difference of temperature between the lateral walls will be the control parameter.

The boundary conditions for the velocity and temperature fields are

$$
\begin{aligned}
& \mathbf{v}=\mathbf{0} \text { on } \partial \Omega, \quad \Theta=0 \text { on } x=0,1, \quad \text { and } \\
& \partial_{y} \Theta=0 \text { on } y=0, \Gamma .
\end{aligned}
$$

Equations (2) and (3) are rewritten in terms of a stream function, $\psi$, related to the velocity field by $\mathbf{v}=$ $\left(\partial_{y} \psi,-\partial_{x} \psi\right)$. They become

$$
\begin{aligned}
& \partial_{t} \Delta \psi-J(\psi, \Delta \psi)=\operatorname{Pr} \Delta^{2} \psi+\operatorname{Ra} \operatorname{Pr}\left(1-\partial_{x} \Theta\right), \\
& \partial_{t} \Theta-J(\psi, \Theta)=\Delta \Theta+\partial_{y} \psi,
\end{aligned}
$$


with $J(f, g)=\partial_{x} f \partial_{y} g-\partial_{y} f \partial_{x} g$, and the boundary conditions are

$\psi=\partial_{x} \psi=0$ on $y=0, \Gamma$, and $\psi=\partial_{y} \psi=0$ on $x=0,1$

$\Theta=0$ on $x=0,1$, and $\partial_{y} \Theta=0$ on $y=0, \Gamma$.

In this way the incompressibility condition is identically fulfilled, and the number of unknowns is reduced.

System (6)-(9) is $\mathcal{Z}_{2}$ equivariant. It remains invariant under the center-symmetry

$$
\mathcal{S}:(t, x, y, \psi, \Theta) \rightarrow(t, 1-x, \Gamma-y, \psi,-\Theta) .
$$

As said in Sec. I, the basic flow of this system is a single vortex, which fills the domain (see Fig. 1a. It is invariant by $\mathcal{S}$, i.e.

$$
\begin{aligned}
& \psi(t, 1-x, \Gamma-y)=\psi(t, x, y), \\
& \Theta(t, 1-x, \Gamma-y)=-\Theta(t, x, y),
\end{aligned}
$$

which also means

$$
\begin{aligned}
& u(t, 1-x, \Gamma-y)=-u(t, x, y), \\
& v(t, 1-x, \Gamma-y)=-v(t, x, y) .
\end{aligned}
$$

This symmetry is broken at some of the Hopf bifurcations found on the branch of steady flows.

To obtain the numerical solutions, the functions $\psi$ and $\Theta$ are approximated by a pseudo-spectral collocation method on a mesh of $n_{x}$ horizontal and $n_{y}$ vertical Gauss-Lobatto points. A resolution of $n_{x} \times n_{y}=40 \times 140$ was used. This means a total of 11200 degrees of freedom. However, in the preliminary tests of the code, other grids were also used to ensure that $40 \times 140$ points were enough to attain a dynamics independent of the mesh size. The spatial operators are then transformed into matrices operating on the values of the functions at the collocation mesh points. Their actions are calculated by means of matrix-matrix products using a highperformance implementation of the DGEMM subroutine of the BLAS library [17]. The stiff system of ordinary differential equations obtained after the spatial discretization is integrated by means of high-order semi-implicit BDF-extrapolation formulas as described, for instance, in Ref. [18].

The branches of steady and periodic solutions were calculated by mean of continuation methods based on the Newton-Krylov techniques proposed in Refs. [19-21], with the linear systems solved by iterative methods. Let $X=\left(\psi_{i j}, \Theta_{i j}\right)$ be a vector containing the values of $\psi$ and $\Theta$ at the collocation points, and $\varphi(t, X, R a)$ the solution of the discretization of the system (6)-(9), at time $t$, for an initial condition $X$ at $t=0$, and for a fixed value of the parameter $R a$. A point $X$ on a periodic orbit, and its period $T$ are solutions of the system

$$
\begin{aligned}
X-\varphi(T, X, R a) & =0, \\
f(X) & =0,
\end{aligned}
$$

where $f(X)=0$ is a phase condition which selects one of the points on the periodic orbit. We have used a Poincaré condition, i.e., the equation of a hyperplane normal to the previously computed cycle. The system defines, by letting $R a$ as a free parameter, a curve of solutions to which continuation methods are applied. As a byproduct, one obtains a method to compute equilibria. They are solutions of $X-\varphi(T, X, R a)=0$ for any value of $T$, which is now a parameter to be fixed. A characteristic time of the problem can be used. In our case it was of the order of the period of the PO of the problem. The phase condition is now not required. In this way the need of writing two different codes for fixed points and $\mathrm{PO}$, and the requirement of a good preconditioner to accelerate the convergence of the linear solver in the case of equilibria are avoided.

\section{STEADY FLOW AND ITS STABILITY}

The branch of steady flows was first computed up to $R a=6 \times 10^{6}$. Since convection is present for any value of $R a$, no matter how small, the continuation was started with the trivial state, $\Psi=0$ and $\Theta=0$, for $R a=0$. The steady flow loses stability in a Hopf bifurcation at $R a=306192.6$. In order to check the code, the critical parameters for the appearance of oscillatory flows were compared with those given in Ref. [15], where primitive variables to formulate the problem and a code for the continuation of fixed points were used. Some of them are shown in Table I. They were found by inverse polynomial interpolation of degrees from one to a maximal value which oscillates from four to ten, depending on the position of the bifurcation points. To facilitate the comparison, all the frequencies are expressed in thermal units taking into account that $\omega_{N S}=\sqrt{\operatorname{RaPr}} \omega_{X Q}$, where the subscript $N S$ refers to the adimensionalization of the present paper and $X Q$ to that of Ref. [15]. With any of the two resolutions shown, the relative errors of the critical Rayleigh numbers, $R a_{c}^{i}, i=1,2,3$, and frequencies $\omega_{c}^{i}, i=1,2$, are below $0.01 \%$, and only $\omega_{c}^{3}$ differs $0.5 \%$. The critical eigenfunctions at these points have also been compared, and can be seen in Ref. [15] and in Fig. 1b-d. Figure 1a shows the contour plots of $\psi$ (left) and $\mathcal{T}$ (right) of the steady solution at $R a=306147.31$. The next three pairs correspond to the real part of the critical eigenfunctions at the first three bifurcations. The left and right plots of each pair show $\psi$ and $\Theta$, respectively.

Seven more Hopf bifurcations have been detected up to $R a=6 \times 10^{6}$. The next three are located at $R a_{c}^{4}=368936.3, R a_{c}^{5}=413526.3$ and $R a_{c}^{6}=466369.1$, with frequencies $\omega_{c}^{4}=1063.4903, \omega_{c}^{5}=1189.4200$ and $\omega_{c}^{6}=1329.6705$. The corresponding eigenfunctions are shown in Fig. 1e-g. As can be seen, the second, fourth and sixth keep the center-symmetry of the steady flow, and the bifurcations give rise to the appearance of $\mathrm{PO}$ that are symmetric at any instant of time ( $F$-cycles). 
TABLE I. Comparison of the critical Rayleigh number and frequency of the first three Hopf bifurcations found on the basic branch of steady solutions with those reported in Ref. [15]. The parameters are $\operatorname{Pr}=0.71$ and $\Gamma=8$, and the frequencies are expressed in thermal units, $d^{2} / \kappa$.

\begin{tabular}{lccccccc}
\hline & $n_{x} \times n_{y}$ & $R a_{c}{ }^{1}$ & $\omega_{c}{ }^{1}$ & $R a_{c}{ }^{2}$ & $\omega_{c}{ }^{2}$ & $R a_{c}{ }^{3}$ & $\omega_{c}^{3}$ \\
\hline XQ02 & $32 \times 120$ & 306180.6 & 796.8574 & 311164.6 & 862.4621 & 333899.6 & 957.3020 \\
NS16 & $32 \times 128$ & 306199.1 & 796.8833 & 311176.2 & 862.4814 & 333908.6 & 953.4198 \\
XQ02 & $40 \times 140$ & 306191.6 & 796.8732 & 311169.8 & 862.4722 & - & - \\
NS16 & $40 \times 140$ & 306192.6 & 796.8746 & 311170.2 & 862.4730 & 333899.4 & 953.4063 \\
\hline
\end{tabular}

The first, third and fifth are antisymmetric, and the bifurcated branches of PO retain the following spatiotemporal symmetry

$$
\begin{aligned}
& \psi(t, 1-x, \Gamma-y)=\psi(t+T / 2, x, y), \\
& \Theta(t, 1-x, \Gamma-y)=-\Theta(t+T / 2, x, y),
\end{aligned}
$$

which also means

$$
\begin{aligned}
& u(t, 1-x, \Gamma-y)=-u(t+T / 2, x, y), \\
& v(t, 1-x, \Gamma-y)=-v(t+T / 2, x, y) .
\end{aligned}
$$

These orbits are said to be symmetric cycles ( $S$-cycles).

The spatial structure of the eigenfunctions consists in vortices of width not larger than $d / 2$, attached to the lateral walls. Their number depends on the eigenfunction. For each set of eigenfunctions sharing the same symmetry, it increases with the corresponding $R a_{c}$. By comparing the contour plots of the left Figs. $1 \mathrm{~b}, \mathrm{~d}$ and $\mathrm{f}$ on the one side and $\mathrm{c}$, e and $\mathrm{g}$ on the other, it can be seen that the additional vortices appear in the center of the cavity, and they are weak. By looking at how they evolve during the critical period $T_{c}=2 \pi / \omega_{c}$, it is seen that they grow as they travel upwards in the left side, and downwards in the right. When they reach the corner they slow down, and their intensity diminishes. Therefore, the instability of the steady flow gives rise to oscillations of small amplitude that propagate in the thin lateral boundary layers, upwards near the hot wall and downwards near the cold one. The number of wavelengths in each side is given by the number of pairs of vortices of the eigenfunction.

\section{PERIODIC ORBITS AND THEIR STABILITY}

As said before, the stable and unstable PO are computed by Newton-Krylov continuation methods. Their stability is analyzed by means of the ARPACK package, based on Arnoldi algorithms (see [22]). The leading spectra (Floquet multipliers of largest modulus) are calculated from the bifurcation points up to $R a=6 \times 10^{5}$ or to $R a$ values from which some multipliers seem to separate definitively from the unit circle (see the last value of each branch in Table II). The bifurcations found are of Neimark-Sacker type, except two of them which are period-doublings (see below for more details).

Figure 2 shows a bifurcation diagram of the PO emerging at the Hopf bifurcations of the steady solutions. A
TABLE II. For $i=0$, critical Rayleigh number, $R a_{c}^{0} \times 10^{-5}$, and frequency $\omega_{c}^{0}$ of the points where the PO bifurcate from the steady state. The capital letters $S$ or $F$ beside the corresponding values state if the $\mathrm{PO}$ arising from this point are $S$-cycles or $F$-cycles, respectively. For $i=1 \cdots 9, R a_{c}^{i} \times 10^{-5}$ and argument of the multiplier of positive imaginary part, $\theta_{c}^{i}$,

\begin{tabular}{|c|c|c|c|}
\hline$i /$ Branch & First & Second & \\
\hline 0 & $(3.0619,796.875) S$ & $(3.1117,862.473)$ & \\
\hline 1 & $(3.3041,0.46473) \circ$ & $(3.1296,0.43048)$ & - \\
\hline 2 & $(3.6271,0.90192) \circ$ & $(4.0436,0.40636)$ & o \\
\hline 3 & $(4.0219,1.29587) \circ$ & $(4.1017,0.82291)$ & ○ \\
\hline 4 & $(4.2913,1.68254) \circ$ & $(4.3193,1.22987)$ & o \\
\hline 5 & $(4.7206,2.03323) \circ$ & $(4.5202,1.65050)$ & o \\
\hline 6 & (5.1987, 2.40926) & $(4.9887,2.10466)$ & ○ \\
\hline 7 & $(5.9614,2.79964) \circ$ & $(5.1067,4.30890)$ & $\bullet$ \\
\hline 8 & & $(5.1827,3.14159)$ & o \\
\hline 9 & & $(5.2156,2.78589)$ & $\circ$ \\
\hline$i$ /Branch & Third & Fourth & \\
\hline 0 & $(3.3390,953.406) S$ & $(3.6894,1063.49)$ & $\bar{F}$ \\
\hline 1 & $(3.4098,0.80539) \bullet$ & $(3.8052,1.12536)$ & • \\
\hline 2 & $(3.4450,0.39057) \bullet$ & $(3.8641,0.75326)$ & • \\
\hline 3 & $(5.3385,2.97307) \circ$ & $(3.9003,0.35041)$ & $\bullet$ \\
\hline 4 & $(5.6356,2.67361) \circ$ & $(4.7024,1.25649)$ & ○ \\
\hline 5 & $(5.6390,2.38747) \circ$ & $(5.0012,1.68881)$ & ○ \\
\hline 6 & $(5.8189,2.05750) \circ$ & $(5.4945,2.16562)$ & ○ \\
\hline 7 & & $(5.7234,3.14159)$ & ○ \\
\hline 8 & & $(5.9320,2.20893)$ & $\bullet$ \\
\hline$i /$ Branch & Fifth & Sixth & \\
\hline 0 & $(4.1353,1189.42) S$ & $(4.6637,1329.67)$ & $\bar{F}$ \\
\hline 1 & $(4.2601,1.39730) \bullet$ & $(4.8069,1.61226)$ & • \\
\hline 2 & $(4.4056,1.06144) \bullet$ & $(5.0218,1.32725)$ & $\bullet$ \\
\hline 3 & $(4.4181,0.70899) \bullet$ & $(5.0424,0.30156)$ & $\bullet$ \\
\hline 4 & $(4.4484,0.32045) \bullet$ & $(5.0654,0.67773)$ & • \\
\hline 5 & $(4.8012,1.14020) \circ$ & $(5.2305,1.38284)$ & ○ \\
\hline 6 & $(4.8812,1.51345) \circ$ & $(5.5935,1.78611)$ & o \\
\hline 7 & $(5.2094,1.93842) \circ$ & $(5.8085,2.16565)$ & ० \\
\hline 8 & $(5.4450,2.34616) \circ$ & $(5.9314,2.51597)$ & o \\
\hline 9 & $(5.7200,2.73154) \circ$ & & \\
\hline
\end{tabular}
at the bifurcation points of the branches of PO. The symbols $(\bullet / \circ)$ indicate whether the critical multiplier gets in or out the unit circle, respectively.

schematic diagram illustrating the connections between unstable PO and the final stable states is also included further in this section. The periods on each branch of PO, $T_{i}$, for $i=1, \cdots, 6$, are plotted versus the Rayleigh number, $R a$, starting at the critical points. The top (black online) curve is the first bifurcated branch, and the bottom (brown online) the sixth. Solid and dashed lines 


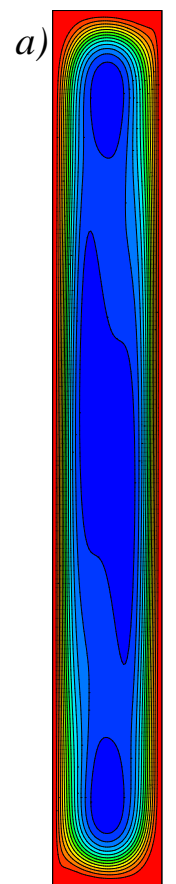

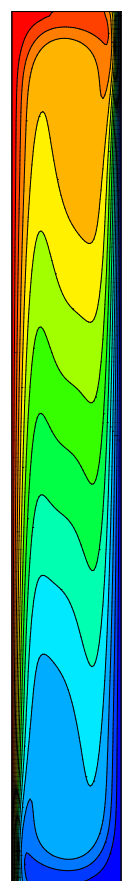

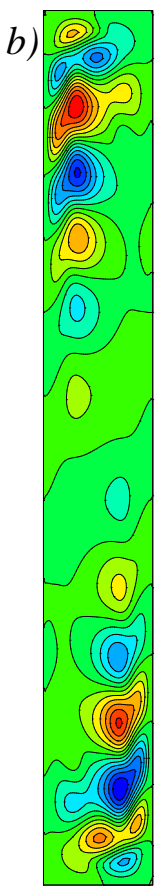

e)
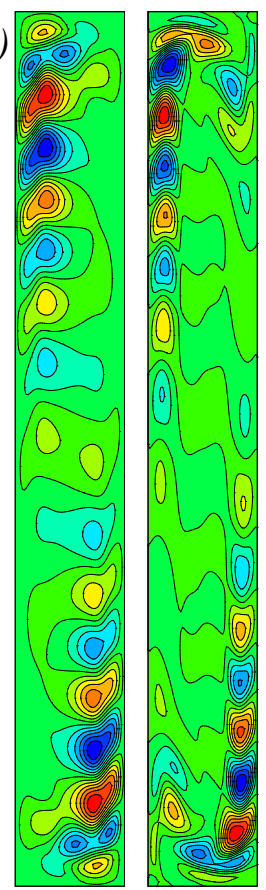

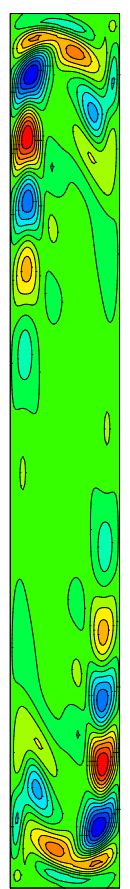

f)
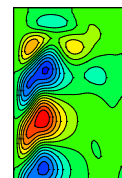

0

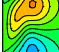

(Q)
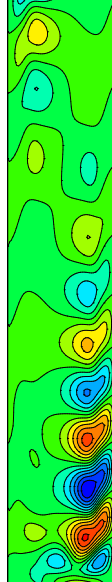

c)
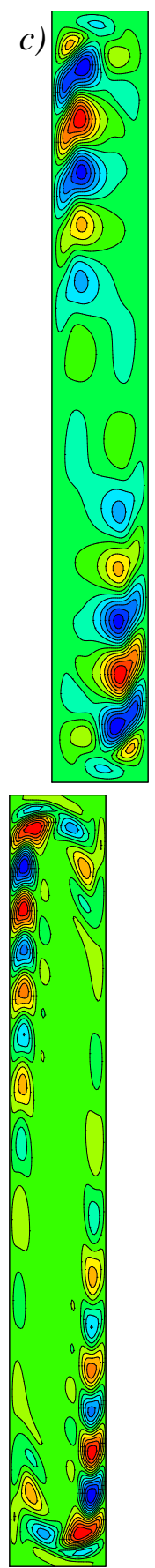

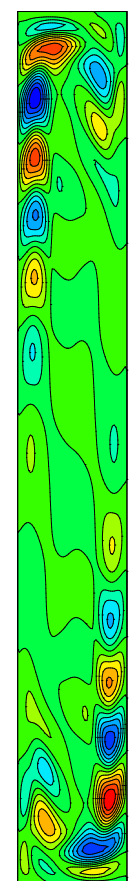

$g)$
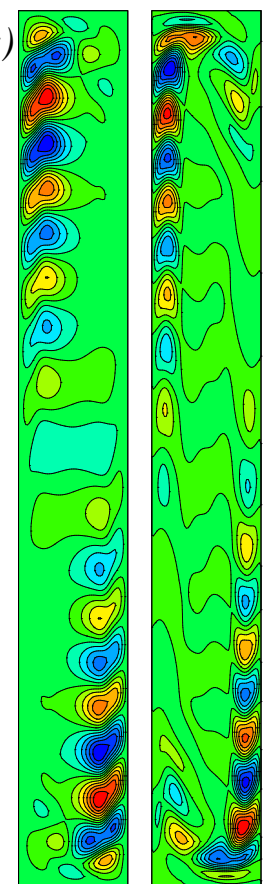

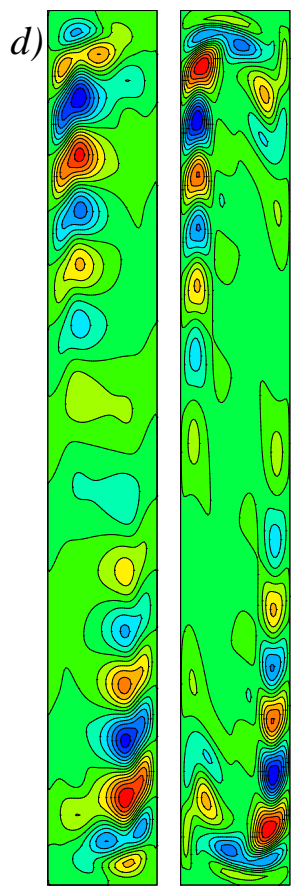

FIG. 1. (a) Stream function, $\psi$, and temperature, $\mathcal{T}$, of a stable steady flow at $R a=306147.31$, and stream function and perturbation of the temperature, $\Theta$, of the real part of the critical eigenfunctions at the (b) first, (c) second, (d) third, (e) fourth, (f) fifth and (g) sixth Hopf bifurcation points on the steady branch of solutions. Online, red means positive (hot) and blue negative (cold).

mean stable and unstable solutions, respectively. Only the first branch of periodic solutions is stable from the bifurcation point. Each one of the following has, initially, a pair of complex-conjugate multipliers outside the unit circle more than the preceding. This means, for instance, that the sixth has, at its origin, five pairs of unstable multipliers. However, from the second to the fifth branch, they are very close to the unit circle, and when $R a$ is increased they move back and stabilize the orbits. The initial unstable segment of the second curve (red online) is so short that cannot be clearly seen in the figure. Figure 3 contains some spectra of cycles on the fifth branch, illustrating the evolution of the multipliers. They remain so close to the unit circle that it is difficult to see in the 


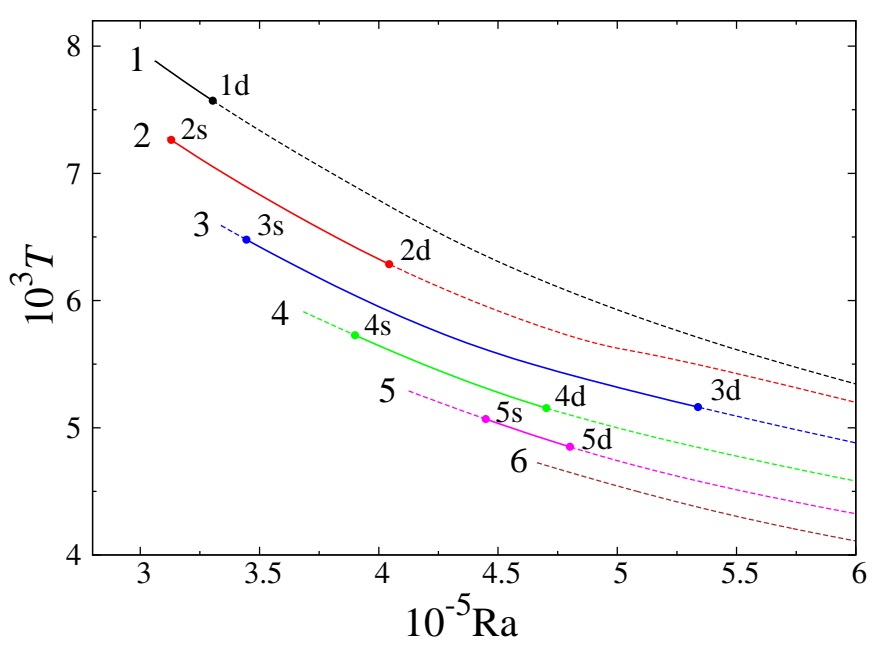

FIG. 2. Period, $T$, versus the Rayleigh number, $R a$, of the first six branches of PO bifurcating from the steady state. Solid lines mean stable solutions and dashed lines unstable. The full circles and the corresponding labels indicate the points where the PO stabilize (is) and destabilize (id), $i=1 \cdots 5$.

plots if some of them are inside or outside. Therefore, the multipliers that are outside are marked with full circles (and red online). The spectrum of Fig. 3e corresponds to a stable solution.

The rows of Table II labeled $i=0$ contain the critical values of the bifurcation points where the PO arise, and their type of global symmetry (see figure caption). The other rows show the critical Rayleigh numbers, $R a_{c}^{i} \times 10^{-5}, i=1 \cdots 6$, and the argument of the critical multipliers of positive imaginary part, $\theta_{c}^{i}$, at the bifurcation points found on the branches of Fig. 2, in the above mentioned range. Full/empty circles beside a pair $\left(R a_{c}^{i}\right.$, $\theta_{c}^{i}$ ) mean that the critical complex multipliers get in/out of the unit circle, respectively. Therefore, the second, third, fourth, and fifth branches stabilize at $3.1295 \times 10^{5}$, $3.4450 \times 10^{5}, 3.9003 \times 10^{5}$ and $4.4484 \times 10^{5}$, respectively, and are stable up to $4.0436 \times 10^{5}, 5.3384 \times 10^{5}$, $4.7024 \times 10^{5}$ and $4.8012 \times 10^{5}$. The sixth does not become stable because only the first four pairs of Floquet multipliers enter the unit circle. The second in crossing is the first that increases its modulus and crosses out again. Notice also that the phase of the eighth critical multiplier on the second branch of PO, and the seventh on the fourth, is $\pi$. This means that they are real and cross the unit circle by -1 , therefore these transitions are the only two period-doubling bifurcations found. According to bifurcation theory, $S$-cycles cannot have a simple multiplier $\mu=-1$ (see [23]).

The first branch is stable only up to $3.3041 \times 10^{5}$. It was checked that the stable periodic orbit calculated in the MIT Conference (see introduction) for $3.4 \times 10^{5}$ belongs to the second branch of Fig. 2, by comparing the averaged
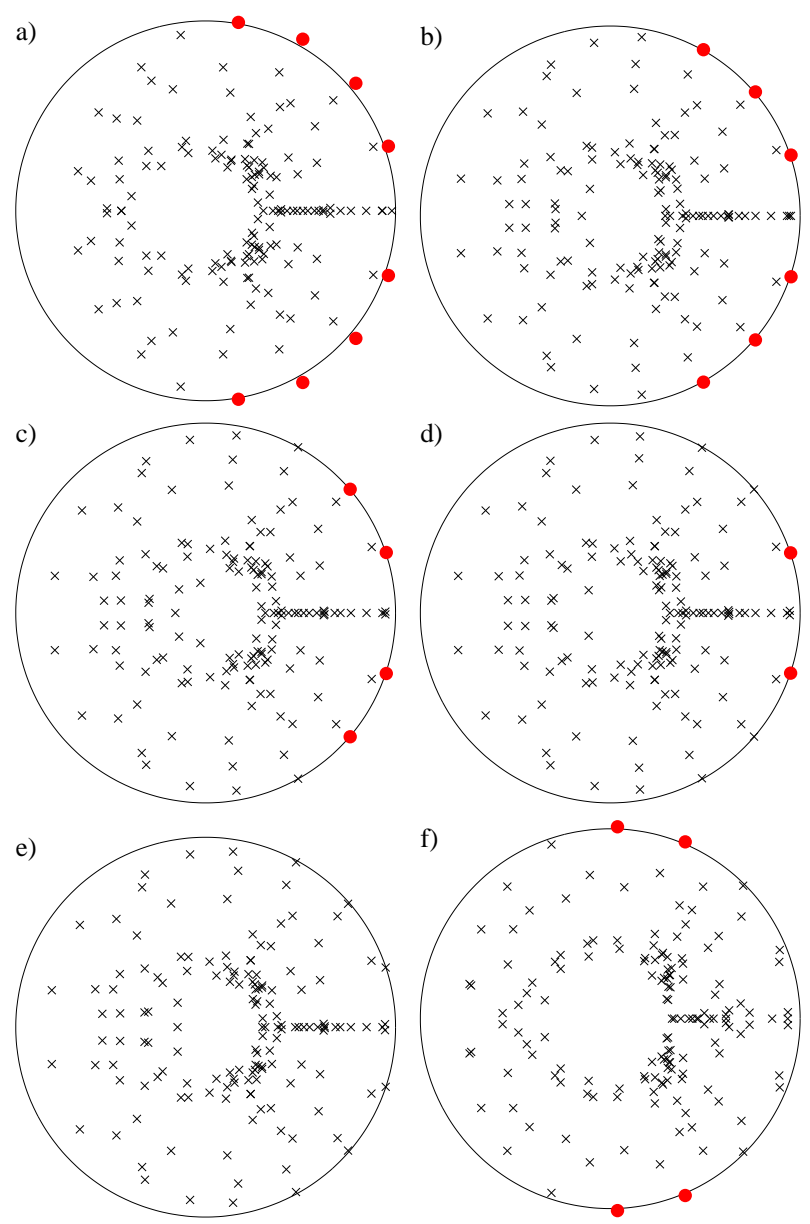

FIG. 3. One hundred and fifty first Floquet multipliers of orbits taken on the fifth branch of PO at (a) 424068.51, (b) 438800.39, (c) 440855.31, (d) 442859.91, (e) 446238.27 and (f) 500000.00. The full circles (red online) indicate the multipliers that are outside the unit circle (solid black curve).

heat flux transport

$$
\overline{\left[\partial_{x} \mathcal{T}\right]}=\frac{1}{T \Gamma} \int_{0}^{T} \int_{0}^{\Gamma} \partial_{x} \mathcal{T} d y d t
$$

across the vertical section $x=1$, and the period of the orbit of the benchmark, with those of Fig. 2. It was found that ${\overline{\left[\partial_{x} \mathcal{T}\right]}}_{x=1}=4.579460$ and $T=6.943542 \times 10^{-3}$, which agree very well with the values of Table III of Ref. [3], mainly with those of the last row corresponding to the calculations by S. Xin and P. Le Quéré, considered by the own authors of the comparison as the reference solution. The maximal deviations found for ${\overline{\left[\partial_{x} \mathcal{T}\right.}}_{x=1}$ and $T$, with respect to the results contributed by the participants in the study, are $2 \%$ and $4 \%$, and the minimal $0 \%$ and $10^{-3 \%}$, respectively.

Figure 4 shows a time evolution of a stable symmetric periodic solution during a period $T$ (see also [24]). Contour plots separated by $T / 2$ are related by the centersymmetry defined by Eqs. (15) and (16), for instance those corresponding to $t=0$ and $T / 2, t=T / 8$ and $5 T / 8$, 

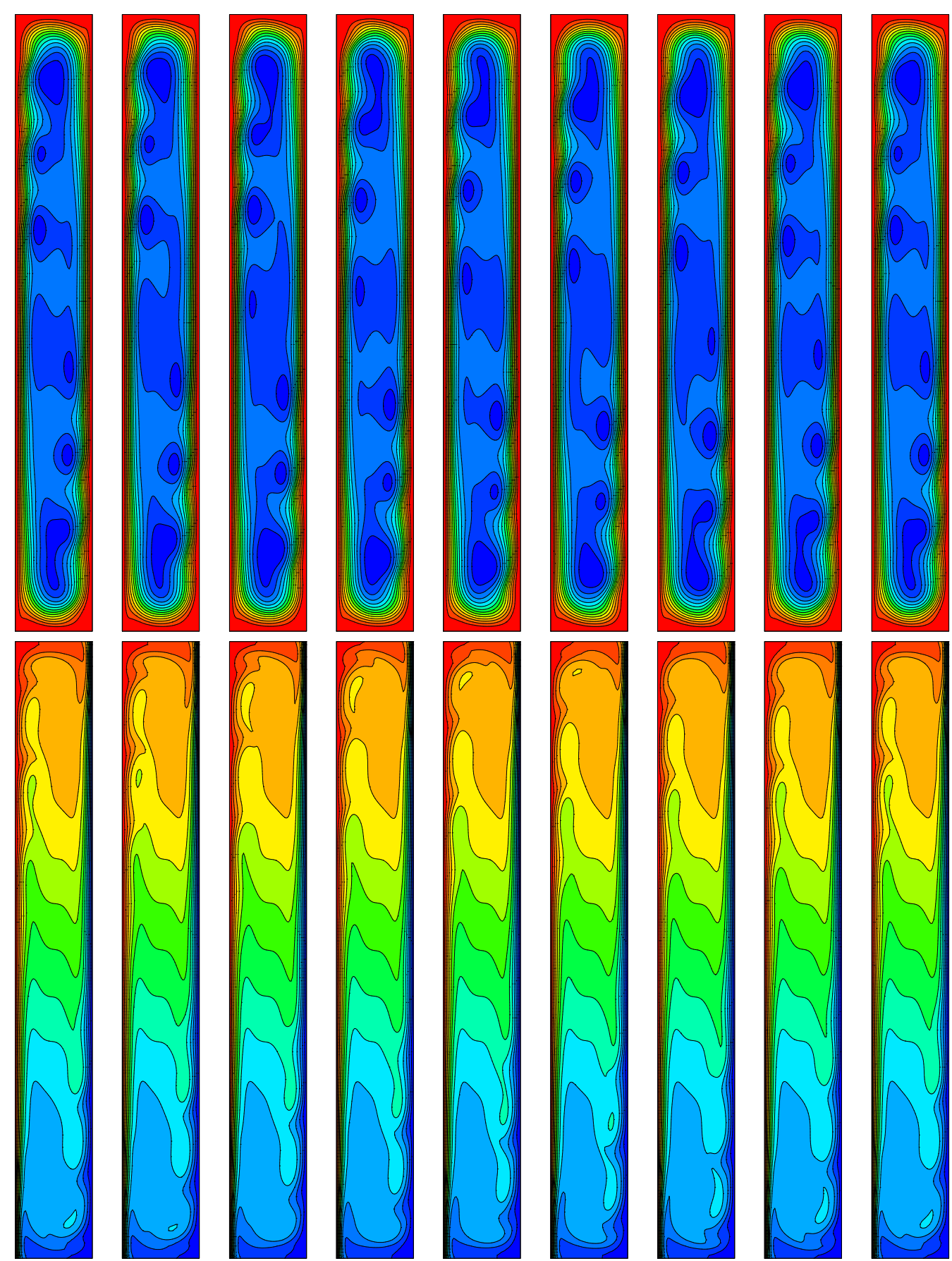

FIG. 4. Snapshots of the stream function (upper row) and the temperature of a stable $S$-cycle of the third branch of Fig. 2, taken at $t=0, T / 8, \cdots, 7 T / 8, T . R a=5 \times 10^{5}$. Dark grey means minimal values of $\psi$ and extreme values of $\mathcal{T}$. (Online, dark blue means minimal values of $\psi$, and red/blue means hot/cold fluid).

and so on. As said before the periodic flow consists in the propagation of a wave of small amplitude in the boundary layer, upwards in the left side of the domain, and downwards in the right. The vortices/cells generated in the middle of the box deform the boundary layer, but since they weaken near the corners the hottest and the coldest fluid do not mix much. The advection transports small bubbles of cold/hot fluid upwards/downwards, but the thermal diffusivity is large enough to homogenize the temperature with that of the surrounding before the bubbles turn to the other side and start to descend dragged by the general circulation. In this way the nearly horizontal stratification of the steady state is only slightly perturbed.

The first five branches of PO of Fig. 2 stabilize and destabilize via Neimark-Sacker bifurcations. The appear- 
ance of a second frequency generates in the flow a modulation of the wave traveling along the boundary layer. Its amplitude, extend and wavelength depend on the branch of PO and the secondary bifurcation considered. To try to find out if these bifurcations are sub or supercritical, i.e. if the emerging quasiperiodic flows are stable or unstable, system (6)-(9) was integrated with initial conditions selected very near the bifurcation points, until the trajectories reached an attractor. Then the frequency of the initial periodic orbit was compared with the frequency/ies of the final solution. The connections between the initial and final states are indicated by vertical arrows in the sketch of the bifurcation diagram of Fig. 5. The bifurcations labeled as $1 d, 2 d, 4 s, 5 s$ and $5 d$ in Fig. 2 are subcritical. In the first case, taking as initial conditions unstable $\mathrm{PO}$ at $R a \gtrsim 3.31 \times 10^{5}$, the stable $\mathrm{PO}$ of the second branch are reached after a transient. The same occurs in the second case $(2 d)$, for $R a \gtrsim 4.05 \times 10^{5}$, the final states being stable PO of the third branch. If an initial condition is taken at $R a=389814.62$, before point $4 s$, a stable quasiperiodic orbit of frequencies $\nu_{1}=174.54$ and $\nu_{2}=9.7847$ is reached. The first frequency is very close to that of the periodic orbit at the bifurcation point, therefore the bifurcation must be subcritical. The same happens if initial conditions are taken at $R a=444759.95$, near bifurcation $5 s$. The frequencies of the stable quasiperiodic orbit are now $\nu_{1}=197.26$ and $\nu_{2}=10.089$. Finally, the time integration with an initial condition at $R a=481898.16$, after the point $5 d$, converges to a three-frequency torus bifurcated from the fourth branch. The frequencies are $\nu_{1}=196.78, \nu_{2}=39.006$ and $\nu_{3}=1.8437$. On the other hand, the time integrations of unstable PO near points $3 d$ and $4 d$ lead to the corresponding bifurcated stable quasiperiodic orbits, and those of unstable PO before points $2 s$ and $3 s$ lead to the stable first and second branches of PO, respectively, so both bifurcations give rise to unstable supercritical quasiperiodic orbits.

According to equivariant dynamical systems theory [23], the tori arising from the Neimark-Sacker bifurcations of the $F$ or $S$-cycles are invariant by the original symmetry $\mathcal{S}$ of Eqs. (6)-(9). This means that if $X$ is a point on one of these tori, then also is $\mathcal{S} X$. To check this and what happens with the stable threetori (see Fig. 5), we proceed as in Refs. [25, 26]. A trajectory is started with an initial condition $X(0)$ on a torus, obtained after eliminating the transients by a long time integration. The relative distance functions $d(t)=\|\mathcal{L} X(0)-X(t)\| /\|X(t)\|$ are then computed for the linear transformations $\mathcal{L}=\mathcal{I}, \mathcal{I}$ being the identity, and $\mathcal{L}=\mathcal{S}$. They are represented in Fig. 6a with solid and dotted lines (black and red online), respectively, for values below 0.01, and for a two-dimensional torus at $R a=485000$. It bifurcates from the segment of stable PO of the fourth branch (see Table II and Figs. 2 and 5). A detail of the FFT of one component of the trajectory is shown in Fig. 6b. The first plot indicates that the torus is invariant with respect to the symmetry $\mathcal{S}$ since the dotted

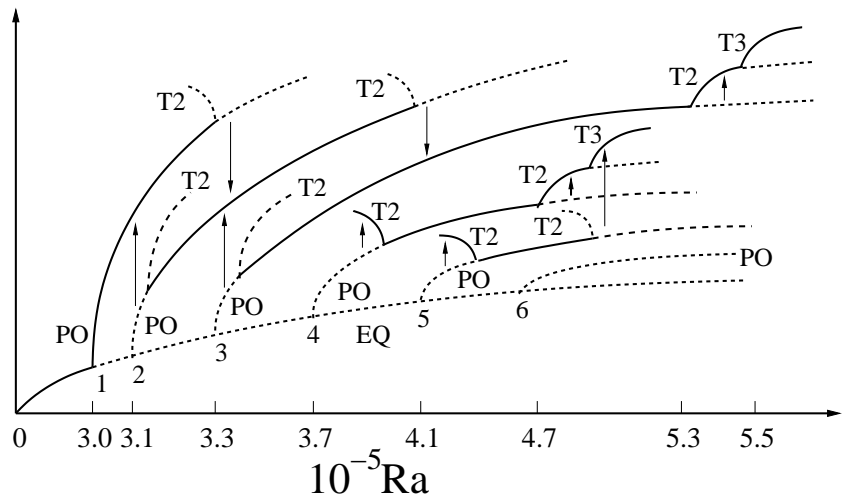

FIG. 5. Sketch of the bifurcation diagram. It shows the type of Neimark-Sacker bifurcations (sub or supercritical) found when the PO lose or gain stability. The lower curve corresponds to the branch of equilibria from which the PO arise. The label EQ means equilibria, and T2 and T3 mean two and three-frequency tori, respectively. The vertical arrows indicate the connections between unstable initial conditions and their final states.

line (red online) peaks near zero every $\Delta t \approx 0.52$ thermal units. More precisely, time intervals oscillating between 0.511 and 0.526 were found. This is also the time between visits to the initial condition (black lines). All the peaks of frequencies $f_{i}$ of Fig. 6b satisfy relations of the form $k_{i}^{0} f_{i}=k_{i}^{1} \nu_{1}+k_{i}^{2} \nu_{2}$, with $\nu_{1}=196.92$ and $\nu_{2}=1.9107$, and integers $k_{i}^{0}, k_{i}^{1}$, and $k_{i}^{2}$. The inverses of the two basis frequencies $T_{1}=5.0782 \times 10^{-3}$ and $T_{2}=0.52337$ are associated, respectively, to the period of the basic PO (see Fig. 2) and to the time between consecutive visits to the initial condition as can be checked in Fig. 6a.

Figure 7 contains the same information but for a threedimensional torus at $R a=500000$, bifurcated from the previous branch of two-dimensional tori after a third Hopf bifurcation. It has been checked that the frequencies of the FFT satisfy $k_{i}^{0} f_{i}=k_{i}^{1} \nu_{1}+k_{i}^{2} \nu_{2}+k_{i}^{3} \nu_{3}$, with $\nu_{1}=202.00, \nu_{2}=2.0085$ and $\nu_{3}=9.8775 \times 10^{-2}$, and integers $k_{i}^{0}, k_{i}^{1}, k_{i}^{2}$, and $k_{i}^{3}$. The first two basis frequencies and its inverses, $T_{1}=4.9505 \times 10^{-3}$ and $T_{2}=0.49787$, are close to that of the previous case. The third quasiperiod, $T_{3}=10.124$, is associated to the new time between consecutive visits to the initial condition or to the transformed point $\mathcal{S} X(0)$, which can be seen in Fig. 7a. Therefore, the three-tori on this branch are still symmetry-invariant.

As the fourth, the third branch of PO loses stability through a Neimark-Sacker bifurcation, and when the quasiperiodic flow destabilizes it also gives rise to a stable three-torus. For instance, at $R a=5.6 \times 10^{5}$ the basis frequencies are $\nu_{1}=199.44, \nu_{2}=11.010$ and $\nu_{3}=1.6265$. This third frequency is one order of magnitude smaller than that found in the three-frequency flows of the fourth branch. We have checked that it is symmetry-invariant. This is the reason why there is no chaos after the appearance of the third frequencies. 

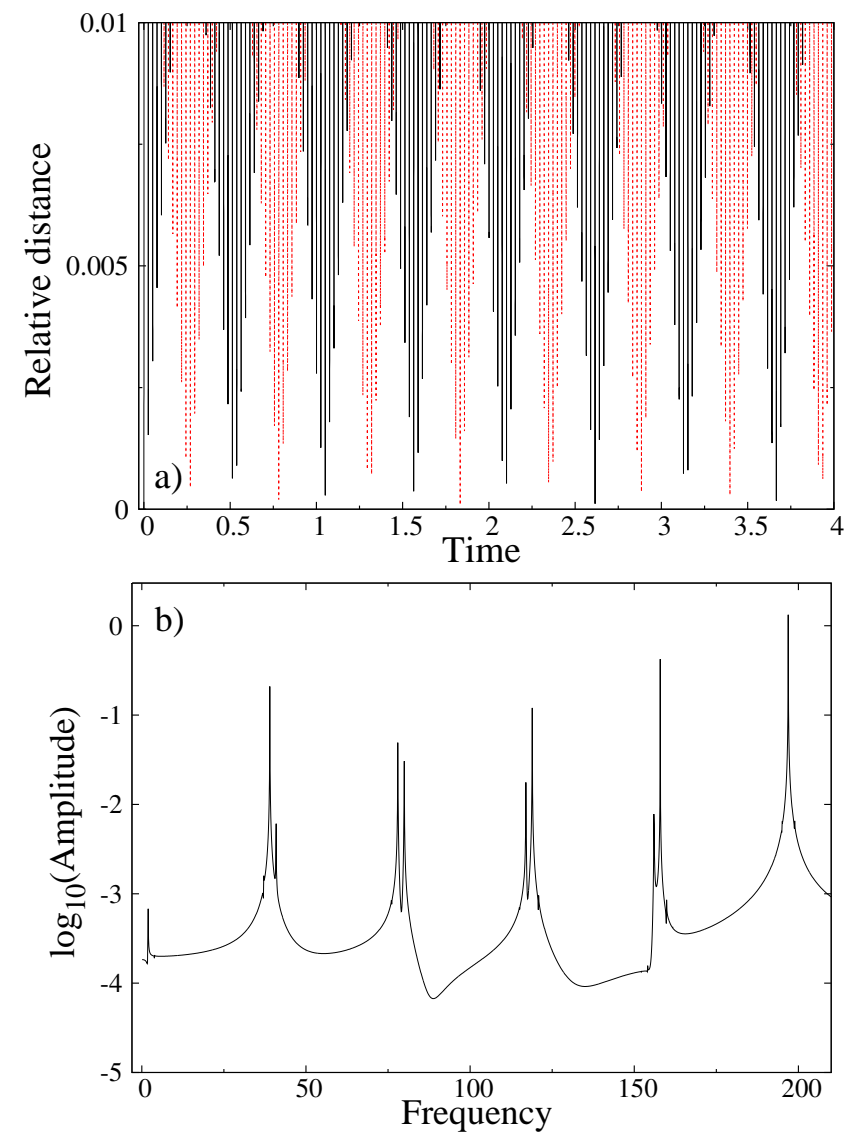

FIG. 6. (a) Relative distances of the transformations $\mathcal{I}$ (black lines online) and $\mathcal{S}$ (red dotted lines online) of an initial condition, $X(0)$, on a torus, bifurcated from the fourth branch of $\mathrm{PO}$, to a generic point of the trajectory $X(t)$ for $R a=485000$, and (b) detail of the FFT of a component of the trajectory.

\section{CONCLUSIONS}

The bifurcation diagram of the branches of periodic flows for a rectangular cavity of aspect ratio eight, differentially heated from the side, has been unfolded. It confirms that the orbits detected previously by time evolution (and other branches detected here) arise directly from the basic steady flow without intermediate turning points or symmetry-breaking bifurcations. Only the first Hopf bifurcation gives rise to stable solutions, but four of the initially unstable branches of PO stabilize via several inverse Neimark-Sacker bifurcations. In addition, all the bifurcation points on the branches of $\mathrm{PO}$ have been determined with precision for a long range of Rayleigh numbers. These results clarify the confusing behavior obtained just using time integrations. In particular, the number of stable periodic flows for a given $R a$ has been stated, and the PO classified according to their spatiotemporal symmetries. Moreover, we have checked, by using direct simulations, that the two subsequent Hopf transitions globally maintain the symmetry.
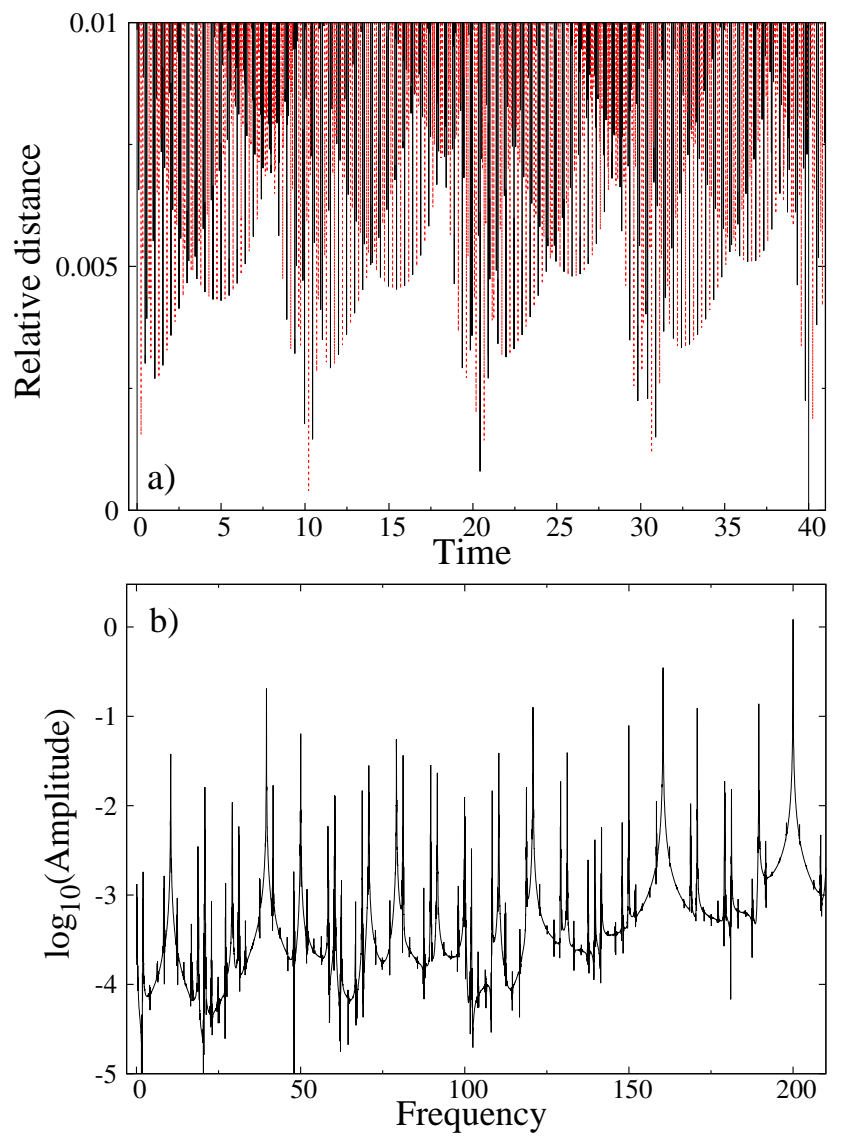

FIG. 7. Same as Fig. 6 but for a three-torus at $R a=500000$.

The spectra of the PO in tall lateral heated domains is not so clustered around the origin as in barotropic thermal convection problems. Although theoretically a good performance of Krylov techniques requires a clustered spectrum [19], and the present configuration is not an ideal case, we have not found any problem in the computation of the PO and their stability. The only difference has been an increase of the number of iterations needed to solve the linear systems and to apply Arnoldi's method.

The modulus of the largest multiplier of the unstable PO is very small, even at the last value computed $(R a=600000)$. For instance, in the first branch it is 1.1932 , but in the fifth it is only 1.0476. Therefore, very long time integrations are needed to pass the transients, which indicates the convenience of using continuation techniques to deal with periodic flows in this problem or in the full three-dimensional version.

\section{ACKNOWLEDGMENTS}

This work has been supported by Spanish MCYT/FEDER grants FIS2013-40674 and FIS201676525-P, and Catalan GENCAT grant 2014-SGR-1145. 
[1] R. Feigelson, ed., 50 years Progress in Crystal Growth. A reprint collection (Elsevier, 2004) p. 269.

[2] K. M. Kim, A. F. Witt, and H. C. Gatos, J. Electrochem. Soc. 119, 1218 (1972).

[3] M. Christon, P. Gresho, and S. Sutton, Int. J. Numer. Meth. Fluids 40, 953 (2002).

[4] S. Xin and P. Le Quéré, Numer. Heat Transfer. Part A 50, 437 (2006).

[5] P. Le Quéré and M. Behnia, J. Fluid Mech. 359, 81 (1998).

[6] K. H. Winters, Int. J. Numer. Meth. Engng 25, 401 (1988).

[7] A. Y. Gelfgat, P. Z. Bar-Yoseph, and A. L. Yarin, J. Fluid Mech. 388, 315 (1999).

[8] A. G. Salinger, R. B. Lehoucq, R. P. Pawlowski, and J. N. Shadid, Int. J. Numer. Meth. Fluids 40, 1059 (2002).

[9] D. Henry and H. Ben Hadid, Phys. Rev. E 76, 016314 (2007).

[10] L. Oteski, Y. Duguet, L. Pastur, and P. Le Quéré, Phys. Rev. E 92, 043020 (2015).

[11] Z. Gao, B. Podvin, A. Sergent, and S. Xin, Phys. Rev. E 91, 013006 (2015).

[12] C.-H. Bruneau and M. Saad, Int. J. Numer. Meth. Fluids 40, 1093 (2002).

[13] D. M. Christopher, Int. J. Numer. Meth. Fluids 40, 1039 (2002).

[14] Y. Guo and K.-J. Bathe, Int. J. Numer. Meth. Fluids 40, 1045 (2002).
[15] S. Xin and P. Le Quéré, Int. J. Numer. Meth. Fluids 40, 981 (2002).

[16] S. Xin and P. Le Quéré, Fluid Dynamics Research 44, 031419 (2012).

[17] K. Goto and R. A. van de Geijn, ACM Trans. Math. Softw. 34, 1 (2008).

[18] F. Garcia, M. Net, B. García-Archilla, and J. Sánchez, J. Comput. Phys. 229, 7997 (2010).

[19] J. Sánchez, M. Net, B. García-Archilla, and C. Simó, J. Comput. Phys. 201, 13 (2004).

[20] J. Sánchez, M. Net, B. García-Archilla, and C. Simó, in Proceedings of the Equadiff-2003 Conference, edited by F. Dumortier, H. Broer, J. Mawhin, A. Vanderbauwhede, and S. V. Lunel (World Scientific, Singapore, 2005) pp. 625-630.

[21] M. Net and J. Sánchez, SIAM J. Appl. Dynam. Systems 14, 674 (2015).

[22] R. B. Lehoucq and D. C. Sorensen, SIAM J. Matrix Anal. Appl. 17, 789 (1996).

[23] Y. A. Kuznetsov, Elements of Applied Bifurcation Theory (Springer, Berlin, 1998).

[24] See the Supplemental Material at http:movie1.gif and http:movie2.gif for two animations of these contour plots.

[25] M. Net and J. Sánchez, Int. J. Bifur. Chaos 15, 3953 (2005).

[26] J. Sánchez, M. Net, and C. Simó, Physica D 239, 123 (2010). 